\title{
One TRPM8 fits all
}

We can sense cold temperatures in different ways: a cool swimming pool on a hot summer's day is pleasant, a cold wind in winter can be painful, and an ice pack can numb the pain of a recently sustained injury. Data from Trpm8-knockout mice have suggested that a single receptor, transient receptor potential melastatin 8 (TRPM8), is involved in the perception of these different types of cold sensation. Takashima et al. now reveal that TRPM8 is expressed in a wide range of neuronal contexts, thus elucidating how a single receptor can convey so many different sensations of cold.

Using mice that expressed green fluorescent protein (GFP) under the control of the Trpm 8 promoter, the authors found that a portion of neurons in the dorsal root ganglia (DRG) and trigeminal ganglia (TG) that expressed GFP also expressed markers for A $\delta$ or $\mathrm{C}$ fibres, which mediate the acute, sharp and slower, dull phases of pain, respectively. In addition, some GFP-expressing neurons in the DRG and TG expressed the pain-related neuropeptide CGRP and TRPV1, which is activated by heat and capsaicin. Together, these findings indicate that a portion of TRPM8-expressing neurons are nociceptors; they also suggest that the remaining population of TRPM8expressing neurons detect innocuous simuli.

The authors then showed that in the teeth of the transgenic mice, GFP was expressed both in dentin, which contains $A \delta$ fibres, and in pulp, which contains $C$ fibres, suggesting that both the first and second stages of cold-induced toothache are mediated by TRPM8-expressing neurons. In skin, TRPM8-expressing neurons terminated in different parts of the dermis and epidermis depending on the skin type, which might account for the fact that hairy skin has a different cold sensitivity from hairless skin. Similarly, in the palate and the tongue, nerve endings of TRPM8expressing neurons were located in different layers of the epithelium, again indicating a role for TRPM8 in diverse cold responses.

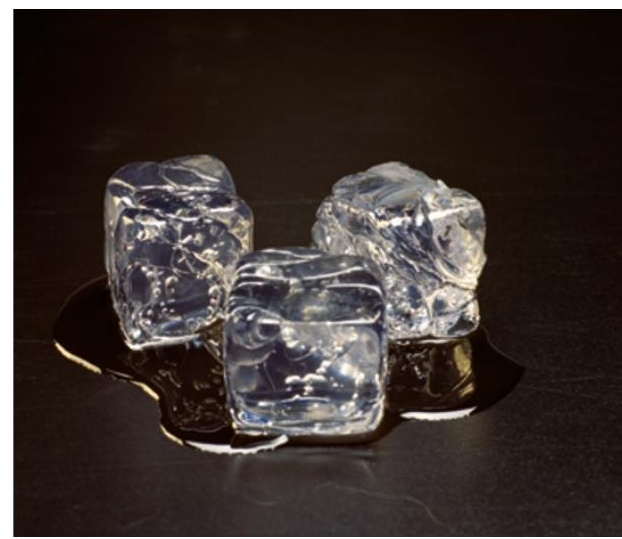

These data demonstrate how a single receptor, through its expression in a variety of sensory-neuron types, can mediate a wide range of cold sensations. Future research to determine whether different TRPM8-expressing neurons have distinct functional properties or are part of discrete neurocircuits will increase our understanding of how the activation of different neurons by the same stimulus leads to such different perceptions.

\section{Leonie Welberg}

ORIGINAL RESEARCH PAPER Takashima, Y. et al Diversity in the neural circuitry of cold sensing revealed by genetic axonal labelling of transient receptor potential melastatin 8 neurons. J. Neurosci. 27, 14147-14157 (2007) 